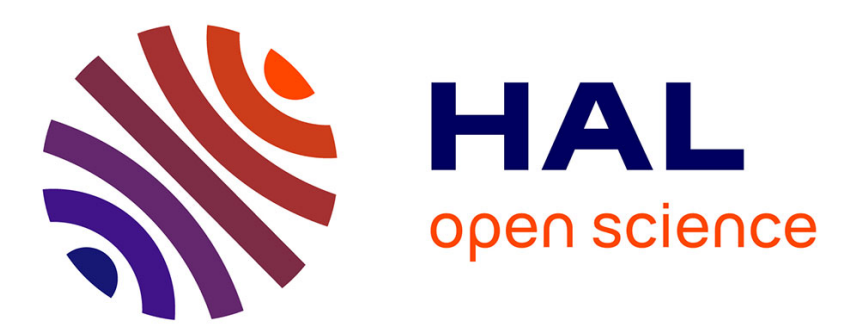

\title{
A Necessary Condition for Waveforms with Better PAPR than OFDM
}

\author{
Marwa Chafii, Jacques Palicot, Rémi Gribonval, Faouzi Bader
}

\section{To cite this version:}

Marwa Chafii, Jacques Palicot, Rémi Gribonval, Faouzi Bader. A Necessary Condition for Waveforms with Better PAPR than OFDM. IEEE Transactions on Communications, 2016, 64 (8), pp.3395-3405. 10.1109/TCOMM.2016.2584068 . hal-01128714v3

\section{HAL Id: hal-01128714 \\ https://hal.science/hal-01128714v3}

Submitted on 30 Jun 2016

HAL is a multi-disciplinary open access archive for the deposit and dissemination of scientific research documents, whether they are published or not. The documents may come from teaching and research institutions in France or abroad, or from public or private research centers.
L'archive ouverte pluridisciplinaire HAL, est destinée au dépôt et à la diffusion de documents scientifiques de niveau recherche, publiés ou non, émanant des établissements d'enseignement et de recherche français ou étrangers, des laboratoires publics ou privés. 


\title{
A Necessary Condition for Waveforms with Better PAPR than OFDM
}

\author{
Marwa Chafii, Member, IEEE, Jacques Palicot, Member, IEEE, Rémi Gribonval, Fellow, IEEE, \\ and Faouzi Bader, Senior, IEEE
}

\begin{abstract}
This paper establishes a necessary condition that must be satisfied by the modulation waveforms of any generalized waveforms for multicarrier (GWMC) system with better peakto-average power ratio (PAPR) than conventional orthogonal frequency division multiplexing (OFDM). GWMC systems include in particular all classical multicarrier modulation systems. As a consequence, we show that OFDM has the best PAPR performance over all GWMC systems that do not satisfy this necessary condition. We also identify an infinite family of GWMC systems with the same PAPR performance as OFDM. To illustrate our results, we present simulations of the PAPR behaviour for different GWMC systems, including some with better PAPR performance than OFDM.
\end{abstract}

Index Terms-Peak-to-Average Power Ratio (PAPR), Orthogonal Frequency Division Multiplexing (OFDM), Generalized Waveforms for Multi-Carrier (GWMC), Fourier Transforms.

\section{INTRODUCTION}

$\mathbf{T}$ He peak-to-average power ratio (PAPR) [1], [2] is a random variable that measures the power variations of the multicarrier modulation signals. The fluctuations of a multicarrier signal envelope generate non-linear distortions when we introduce the signal into the high power amplifier (HPA), due to the non-linearity of the HPA response. To avoid these distortions, an input back-off is needed in order to amplify the signal in the linear area of the HPA. The larger the PAPR, the larger the input back-off introduced, and the smaller the HPA efficiency. The energy consumption of the power amplifier represents $60 \%$ of the total energy consumption in a base station [3]. Therefore, the signal amplitude variations should be reduced in order to reach a better HPA efficiency and minimize the power consumption.

As presented later in the state of the art, several multicarrier modulation systems based on different waveforms have been proposed in the literature as alternatives to conventional orthogonal frequency division multiplexing (OFDM) [4]. In addition to the PAPR, there are several relevant criteria for selecting or designing new waveforms for future multicarrier systems, which include, among others:

- Bit error rate to evaluate reliability of the transmission.

\footnotetext{
M. Chafii, J. Palicot and Faouzi Bader are with the CentraleSupélec/IETR, $35576 \quad$ Cesson-Sévigné, France (email: $\{$ marwa.chafii, jacques.palicot,faouzi.bader $\}$ @ supelec.fr).

R. Gribonval is with Inria Rennes-Bretagne Atlantique, 35042 Rennes Cedex, France (e-mail:remi.gribonval@inria.fr.

This work has received a French state support granted to the CominLabs excellence laboratory and managed by the National Research Agency in the "Investing for the Future" program under reference Nb. ANR-10-LABX-0701. The authors would also like to thank the Region Bretagne, France, for its support of this work
}

- Complexity to ensure feasibility of the system.

- Bandwidth efficiency to ensure a high transmission rate using the available resources.

- Latency to reduce the delay of the transmission.

- Data rate to meet the needs of high-speed applications.

In this paper, we focus on the PAPR criterion, and we investigate the behaviour of the PAPR regarding to the modulation waveforms. It has been proved that the PAPR depends on the waveform used in the modulation [5], [6]. Thus, instead of using PAPR reduction techniques [7]-[11], one can change the PAPR performance by changing the characteristics of the waveform, which gives new insights regarding PAPR reduction. In this work, we show analytically that having a temporal support ${ }^{1}$ strictly less than the symbol period is a necessary condition on alternative waveforms with better PAPR than OFDM ${ }^{2}$.

In fact, we prove that, if the previous necessary condition is not satisfied, i.e if the waveforms have a temporal support larger than or equal to the symbol period, then their PAPR performance cannot be better than that of conventional OFDM. In addition, we identify several multicarrier modulation systems with the same PAPR performance as OFDM. Fig. 9 provides an insight into the conclusions of this work.

\section{A. Paper outline}

Our main contributions are to prove that:

1) Among systems which waveforms have a temporal support larger than or equal to the symbol period:

- The (distribution of the) PAPR is optimal only if the sum of the squared modulus of the individual (multicarrier) waveforms is constant over time. (See (4)).

- An infinite number of GWMC systems are optimal in terms of PAPR performance.

- OFDM is among this class of GWMC systems.

- No better PAPR compared to OFDM is possible, without reducing the temporal support of the waveforms.

2) Signal sets may have better PAPR than OFDM, when the temporal support is limited (e.g. Haar wavelet).

\footnotetext{
${ }^{1}$ The support of a function means here the interval outside which the function is equal to zero.

${ }^{2}$ The study here considers OFDM without guard interval, but the analysis is the same for OFDM with cyclic prefix, since the addition of a cyclic prefix does not give any additional information about the peak power.
} 


\section{B. State of the art}

Several classes of multicarrier modulation systems can be defined [12], [13], based on several characteristics, including whether the modulation is based on the discrete Fourier transform or other transforms, and orthogonal or non-orthogonal schemes. A first distinction can be made:

- Modulations based on the Fourier transform: the modulation scheme can be based on the inverse fast Fourier transform (IFFT) at the transmitter side and the fast Fourier transform (FFT) at the receiver side. After the IFFT block, the signal is filtered with pulse shaping filters. Depending on the selected filter, the resulting signal is orthogonal or nonorthogonal. Hereafter, we provide some examples of such waveforms:

- OFDM is a well known orthogonal scheme based on the Fourier transform and the rectangular filter.

- NOFDM: non-orthogonal frequency division multiplexing

[14] is a multicarrier modulation system that does not have any restriction about the distance between pulses in the time-frequency plane, and the design of the pulse shape, which leads to a better bandwidth efficiency, while the time frequency location and the shape of the pulses for conventional OFDM are strictly defined.

- OFDM/OQAM:(offset quadrature amplitude modulation) [15]-[17] is a filter bank multicarrier (FBMC) [18] system that allows a flexible selection of the pulse shaping filters, such as the isotropic orthogonal transform algorithm (IOTA) [19], the extended Gaussian functions (EGF), the PHYDYAS filter ${ }^{3}$, and the Hermite filters, in order to reduce side lobes without using guard bands in contrast to conventional OFDM. OFDM/OQAM does not use a cyclic prefix.

- GFDM: generalized frequency division multiplexing [20], [21] is a non-orthogonal scheme which allows the use of well-localized filters to avoid out-of-band emissions. The modulation is performed block by block, where each GFDM data block consists of a certain number of carriers and symbols. A cyclic prefix can be added in the GFDM data block, along with tail biting in the pulse shape.

For more details, a taxonomy of multicarrier modulation systems is proposed in [12]. For the different pulse shaping filters, the reader can refer to [13] that defines and gives the analytical expression and characteristics of the most popular prototype filters in the literature.

- Modulations based on other transforms: instead of performing the modulation based on the Fourier transform, other transforms can be used. These are examples reported in the literature:

- The Wavelet Transform is widely used in wireless communication applications. These multicarrier modulation systems can be based on the discrete wavelet transform (DWT) [22] or the wavelet packet transform (WPT) [23].

- The Walsh-Hadamard Transform can be used as a modulation basis for multicarrier modulation systems.

${ }^{3}$ Physical Layer For Dynamic Spectrum Access And Cognitive Radio, more details on http://www.ict-phydyas.org/
In [24], the author introduces the Walsh-Hadamard transform for multicarrier modulation applications, and compares it with the Fourier transform in conventional OFDM. It has been also showed that multicarrier modulation systems based on the Walsh-Hadamard transform are more suitable for optical communications than OFDM at short distance transmission, in terms of computational complexity [25].

- The Discrete Cosine Transform (DCT) has been proposed in the literature [26], [27] as a modulation basis for multicarrier systems. In [28], the multicarrier modulation scheme based on the DCT has been proved better than OFDM in terms of BER under certain channel conditions.

The remainder of this paper is structured as follows. In Section II, we define the generalized waveforms for multicarrier (GWMC) system considered in our derivations, and we formulate the PAPR reduction problem as an optimization problem. The solution of this problem is given in Section III with the whole proof behind. To support the theoretical results, we illustrate some examples of multicarrier modulation systems in Section IV. Finally, Section V concludes the paper and opens new perspectives of the work.

\section{PRoblem Formulation}

\section{A. Notation: the GWMC model}

The GWMC system is a generalization of classical multicarrier modulation systems based on a larger choice of modulation schemes. The GWMC transmitted signal is expressed as

$$
X(t)=\sum_{n \in} \sum_{m=0}^{M-1} C_{m, n} \underbrace{g_{m}(t-n T)}_{g_{m, n}(t)},
$$

where $M$ denotes the number of carriers, $C_{m, n}$ stands for the complex input symbol, time index $n$, modulated by carrier index $m$, and $T$ is the GWMC symbol period. The modulation transform and the pulse shaping filter are jointly modeled by a family of functions denoted by $\left(g_{m}\right)_{m \in \llbracket 0, M-1 \rrbracket}$. Note that the model in (1) includes the single carrier case.

We define the PAPR of the GWMC signal as follows

$$
\begin{aligned}
\text { PAPR } & =\frac{\max _{t \in[0, T]}|X(t)|^{2}}{P_{\text {mean }}}, \\
P_{\text {mean }} & =\lim _{t_{0} \rightarrow+\infty} \frac{1}{2 t_{0}} \int_{-t_{0}}^{t_{0}} E\left(|X(t)|^{2}\right) d t .
\end{aligned}
$$

$E($.$) is the expectation operator. The mean power P_{\text {mean }}$ is defined over an infinite integration time, because our scenario assumes an infinite transmission time, but the observation is limited to a single GWMC symbol.

\section{B. Main assumptions}

The number of carriers is supposed to be $M \geq 8$. This is an assumption made for the validity of the central limit theorem. We assume that $\left(C_{m, n}\right)_{(m \in \llbracket 0, M-1 \rrbracket, n \in \mathbb{Z})}$ are independent and identically distributed, with zero mean and unit variance $\sigma_{C}^{2}$. Let $\left(g_{m}\right)_{m \in \llbracket 0, M-1 \rrbracket} \in L_{I}^{\infty}$, where $L_{I}^{\infty}$ is the space of essentially bounded functions which vanish outside a finite interval $I$. Then we have

$$
\forall m \in \llbracket 0, M-1 \rrbracket \quad \forall t \notin I \quad g_{m}(t)=0 .
$$




\section{Optimization problem associated to PAPR reduction}

As we will soon recall in Section II-E, the PAPR reduction problem can be formulated as the following constrained optimization problem.

\section{The PAPR Optimization Problem.}

$$
\begin{aligned}
& \underset{\left(g_{m}\right)_{m \in \mathbb{N}, M-1 \rrbracket}}{\operatorname{maximize}} \quad \int_{0}^{T} \ln \left(1-\mathrm{e}^{\frac{-\gamma \sum_{m=0}^{M-1}\left\|g_{m}\right\|^{2}}{T \sum_{n \in \mathbb{Z}} \sum_{m=0}^{M-1}\left|g_{m, n}(t)\right|^{2}}}\right) d t, \\
& \text { subject to } A:=\min _{m, t} \sum_{n \in Z}\left|g_{m, n}(t)\right|^{2}>0 .
\end{aligned}
$$

The quantity that we want to maximize in the PAPR optimization problem is equivalent to minimizing the approximation of the complementary cumulative distribution function (CCDF) of the PAPR, subject to the constraint expressed in (2). The CCDF is the probability that the PAPR exceeds a defined value $\gamma$, i.e. $\operatorname{Pr}(\operatorname{PAPR} \geq \gamma)$.

\section{The meaning of condition (2)}

Roughly speaking, (2) means that the translated versions of every carrier $g_{m}$ are overlapping in time, and the waveform $g_{m}$ does not vanish within the symbol period $T$.

If there exists at least an index $m_{0} \in \llbracket 0, M-1 \rrbracket$ such that $g_{m_{0}}$ vanishes in a time interval in the symbol period $T$ and its support is of length at most $T$, then the family of functions $\left(g_{m}\right)_{m \in \llbracket 0, M-1 \rrbracket}$ does not satisfy (2). In this case, we can also say that $g_{m_{0}}$ has a temporal support strictly smaller than the symbol period $T$.

A particular consequence is that $g_{m_{0}}$ is likely to have a larger frequency support and then a worse frequency localization. This is due to the fact that time frequency localization measure is limited by the Heisenberg uncertainty principle ${ }^{4}$.

\section{E. Origins of the optimization problem formulation}

In order to formulate the PAPR optimization problem, we have first derived in [6] an approximation of the CCDF of the PAPR for the GWMC systems. Based on the Lyapunov central limit theorem (CLT), we showed that the distribution of the signal is approximately Gaussian, and then we derived the approximate distribution of the PAPR by considering a simplifying assumption related to the independence of the samples. To apply the Lyapunov CLT, Lyapunov conditions should be verified. For this purpose, we assumed that the modulation functions satisfy the conditions in (2) and $B:=\max _{m, t} \sum_{n \in Z}\left|g_{m}(t-n T)\right|<+\infty$, where the second condition is always satisfied for all the functions belonging to $L_{I}^{\infty}$ space. In practice, all the waveforms should be bounded and have a finite support, therefore they necessarily belong to $L_{I}^{\infty}$, that is why this condition does not appear in the PAPR optimization problem.

It is important to highlight that the assumption of independence of the samples is not always verified in practice,

\footnotetext{
${ }^{4}$ Or sometimes the Heisenberg-Gabor theorem, it states that a function cannot be both time-limited and band-limited (a function and its Fourier transform cannot both have bounded domain). Then, one cannot simultaneously sharply localize a signal in both the time domain and the frequency domain. More details can be found in [29].
}

which makes the derived distribution only an approximation. Simulation results showed that for a large number of carriers, the curve based on the theoretical approximation fits the empirical one. For more details about why we need (2) to satisfy Lyapunov conditions and how we derive the CCDF approximation based on Lyapunov CLT, the reader can refer to the authors work in [6].

\section{MAin TheORETICAL RESUltS}

In this section, the solution of the PAPR optimization problem is presented. The PAPR optimality of conventional OFDM is also proved and discussed. The early work in this context goes back to the study undertaken by A. Skrzypczak et al. for OFDM/OQAM and oversampled OFDM [30], where it has been shown analytically that the PAPR performance for the latest two multicarrier modulation systems based on different pulse shapes is not better than that of conventional OFDM with the rectangular pulse shape.

Based on simulation results, A. Kliks [31] has further noticed that, when simulating the CCDF of the PAPR for the generalized multicarrier (GMC) signal for different pulses, the lowest values are obtained for the rectangular pulse. In the analysis conducted in this paper, we consider the GWMC system, which is a generalization of classical multicarrier modulation systems and based on a larger choice of modulation schemes.

\section{A. Overview of the main results}

Let $H_{0} \approx 0.63$ be the unique solution to the equation $1-2 H_{0}+2 H_{0} \mathrm{e}^{\frac{-1}{H_{0}}}=0$ (see Appendix). For a given GWMC system $\left\{g_{m}\right\}$, we define the "critical" value of $\gamma$ for this system as

$$
\gamma_{\text {crit }}\left(\left\{g_{m}\right\}\right):=\sup _{t \in[0, T]} \frac{T \sum_{m=0}^{M-1} \sum_{n \in}\left|g_{m, n}(t)\right|^{2}}{H_{0} \sum_{m=0}^{M-1}\left\|g_{m}\right\|^{2}} .
$$

Our main technical result is the following proposition, where the proof will be provided in Section III-C.

Property 1. (Sufficient condition for optimality) Assume that $\gamma \geq 1 / H_{0}$ and consider any GWMC system $\left\{g_{m}^{*}\right\}$ satisfying (2) and

$$
\sum_{m=0}^{M-1} \sum_{n \in}\left|g_{m, n}^{*}(t)\right|^{2} \quad \text { is constant over time. }
$$

For this system $\gamma_{c r i t}\left(\left\{g_{m}^{*}\right\}\right)=1 / H_{0}$ has the minimum possible value.

At level $\gamma$, the CCDF of the PAPR of the system $\left\{g_{m}^{*}\right\}$ is lower (corresponding to a better PAPR) than that of any other system $\left\{g_{m}\right\}$ satisfying (2) such that $\gamma \geq \gamma_{\text {crit }}\left(\left\{g_{m}\right\}\right)$.

In other words, the GWMC system $\left\{g_{m}^{*}\right\}$ has globally optimal PAPR performance at level $\gamma$ among all GWMC systems satisfying (2) and $\gamma \geq \gamma_{\text {crit }}\left(\left\{g_{m}\right\}\right)$.

The condition in (4) means that the statistical mean of the instantaneous power of the transmitted signal $E\left(|x(t)|^{2}\right)$ is constant over time.

Corollary 1. (Optimality of conventional OFDM)

Consider $\left\{g_{m}\right\}$ a GWMC system satisfying (2). For any $\gamma \geq$ 
$\gamma_{\text {crit }}\left(\left\{g_{m}\right\}\right)$, this system has PAPR performance at level $\gamma$ no better than that of OFDM, the Walsh-Hadamard system, and of any other GWMC system satisfying (4).

These results will be illustrated in Sections IV-A and IV-B.

Hence, the logical contraposition of Corollary 1 gives the following Theorem.

Theorem. (Necessary condition for improving PAPR performance)

Consider a GWMC system $\left\{g_{m}\right\}$ and $\gamma \geq \gamma_{\text {crit }}\left(\left\{g_{m}\right\}\right)$. If this GWMC system has better PAPR performance at level $\gamma$ than OFDM, then $\left\{g_{m}\right\}$ necessarily violates condition (2).

As explained in Section II-D, the fact that GWMC violates condition (2) means that the temporal support of at least one modulation function must be strictly smaller than the symbol period. Thus, we are led to a trade-off between frequency localization of multicarrier waveforms and PAPR performance. This phenomenon will be illustrated in Section IV-C.

\section{B. Discussion}

The condition $\gamma \geq \gamma_{\text {crit }}\left(\left\{g_{m}\right\}\right)$ satisfied by a GWMC system means that our results are valid for the values of $\gamma$ greater than a threshold value $\gamma_{\text {crit }}\left(\left\{g_{m}\right\}\right)$.

For any GWMC system satisfying (2) such that OFDM or Walsh-Hadamard multicarrier system, we have $\gamma_{\text {crit }} \approx 2 \mathrm{~dB}$. For the weighted cyclic prefix-OFDM system discussed in Section IV-B, we have $\gamma_{\text {crit }} \approx \frac{1.2}{H_{0}}=2.7 \mathrm{~dB}$.

Our analysis does not cover the values of $\gamma$ smaller than $\gamma_{\text {crit }}$. In practice, the PAPR of multicarrier modulation systems is greater than $\gamma_{\text {crit }}$. Thus, this interval does not represent an interval of interest.

\section{Proof}

Replacing the PAPR Optimization Problem with a Simpler Problem. In order to characterize the optima of the PAPR optimization problem, we first show that it can be rewritten in a simpler form. We start by noticing that the functions $\left(g_{m}\right)_{m \in \llbracket 0, M-1 \rrbracket}$ perform the same role and only the $\operatorname{sum} \sum_{n \in \mathbb{Z}} \sum_{m=0}^{M-1}\left|g_{m, n}(t)\right|^{2}$ is involved in the maximized quantity, the maximization can thus be performed over only one non-negative function $G(t)$, such that

$$
G(t)=\sum_{m=0}^{M-1} \sum_{n \in \mathbb{Z}}\left|g_{m, n}(t)\right|^{2} .
$$

Note that (2) implies that $a:=\inf _{t} G(t)>0$. Similarly, $G \in L^{\infty}([0, T]), L^{\infty}$ is the space of essentially bounded functions. Moreover,

$$
\begin{aligned}
\int_{0}^{T} G(\tau) d \tau & =\int_{0}^{T} \sum_{m=0}^{M-1} \sum_{n \in}\left|g_{m, n}(t)\right|^{2} d t \\
& =\sum_{m=0}^{M-1} \sum_{n \in} \int_{n T}^{n T+T}\left|g_{m}(t)\right|^{2} d t \\
& =\sum_{m=0}^{M-1} \int_{-\infty}^{+\infty}\left|g_{m}(t)\right|^{2} d t \\
& =\sum_{m=0}^{M-1}\left\|g_{m}\right\|^{2} .
\end{aligned}
$$

The optimization problem can thus be expressed as

$$
\begin{aligned}
\underset{G \in L^{\infty}([0, T])}{\operatorname{maximize}} & \beta(G)=\int_{0}^{T} \ln \left(1-\mathrm{e}^{\frac{-\gamma \int_{0}^{T} G(\tau) d \tau}{T G(t)}}\right) d t . \\
\text { subject to } & \exists a \text { such that } \\
& G(t) \geq a>0 .
\end{aligned}
$$

Finally, the condition $\gamma \geq \gamma_{\text {crit }}\left(\left\{g_{m}\right\}\right)$ is equivalent to

$$
\sup _{t \in[0, T]} G(t) \leq \gamma H_{0} \int_{0}^{T} G(\tau) d \tau .
$$

Remark 1. Denoting $H(t):=G(T t)$, we have

$$
\begin{aligned}
\beta(G)= & T \int_{0}^{1} \ln \left(1-\mathrm{e}^{\frac{-\gamma \int_{0}^{1} H(\tau) d \tau}{H(t)}}\right) d t \\
=: & T \tilde{\beta}(H), \\
\text { and } \quad & H \geq a>0 .
\end{aligned}
$$

Maximizing $\beta$ with respect to $G \in L^{\infty}([0, T])$ is then equivalent to maximizing $\tilde{\beta}$ with respect to $H \in L^{\infty}([0,1])$.

Moreover the expression of $\tilde{\beta}(H)$ does not change if we multiply the function $H(t)$ by a scalar: for all $\lambda \in \mathbb{R}^{*+}$, we have

$$
\tilde{\beta}(\lambda H)=\tilde{\beta}(H) .
$$

It follows that if the problem in (7) has an optimal solution, then there exists an infinite set of optimal solutions obtained by scaling the first solution.

From Remark 1, we can search the maximizer of $\tilde{\beta}$ under the additional normalization constraint

$$
\gamma \int_{0}^{1} H(\tau) d \tau=1
$$

If $G$ is an optimum of problem (7), then $H(t):=\frac{T G(t T)}{\gamma \int_{0}^{T} G(\tau) d \tau}$ is an optimum of the following optimization problem

$$
\begin{aligned}
\underset{H \in L^{\infty}([0,1])}{\operatorname{maximize}} & \tilde{\beta}(H):=\int_{0}^{1} \ln \left(1-\mathrm{e}^{\frac{-1}{H(t)}}\right) d t . \\
\text { subject to } & H \text { satisfies }(12) \text { and } \exists a \text { such that } \\
& H(t) \geq a>0 .
\end{aligned}
$$

Vice-versa, if $H$ is an optimum of the above problem then $G(t)=H(t / T)$ is an optimum of problem (7). The rest of the study therefore focuses on characterizing the optima of problem (13).

Note that the condition $\gamma \geq \gamma_{\text {crit }}\left(\left\{g_{m}\right\}\right)$ now read simply

$$
\sup _{t \in[0, T]} H(t) \leq H_{0} \text {. }
$$

Theoretical Analysis. We define the following convex subsets of $L^{\infty}$ :

- $\mathcal{F}:=\left\{H:[0,1] \rightarrow \mathbb{R}^{*+}\right.$ such that $\left.\int_{0}^{1} H(\tau) d \tau=\frac{1}{\gamma}\right\}$,

- $\mathcal{F}_{a}:=\mathcal{F} \cap\left\{H:[0,1] \rightarrow \mathbb{R}^{*+}\right.$ such that $\left.H \geq a\right\}$,

- $\mathcal{F}_{+}:=\cup_{a>0} \mathcal{F}_{a}$.

We consider here the optimization problem in (13). To characterize its optima, we first recall the definition of its stationary points.

Definition 1. We say that a function $H^{*} \in \mathcal{F}_{a}$ is a stationary point of $\tilde{\beta}$ defined in (13) if and only if: for any $\varphi \in L^{\infty}([0,1])$ such that

$$
\int_{0}^{1} \varphi(t) d t=0
$$


we have

$$
\left.\frac{d \tilde{\beta}\left(H^{*}+\epsilon \varphi\right)}{d \epsilon}\right|_{\epsilon=0}=0 .
$$

Notice that for all $\varphi$ satisfying (14), the function $H=H^{*}+$ $\epsilon \varphi$ satisfies (12). For a small enough $\epsilon, H=H^{*}+\epsilon \varphi$ also satisfies (10).

The solution of the optimization problem is organized as follows

\section{Lemma 1.}

Let $H_{0}$ be the unique solution to the equation $1-2 H_{0}+2 H_{0} \mathrm{e}^{\frac{-1}{H_{0}}}=0$. For any $\varphi \in L^{\infty}([0,1])$ which satisfies (14), we have: for any $H \in \mathcal{F}_{+}$,

$$
\text { If } \sup _{t} H(t) \leq H_{0}, \quad \text { then }\left.\quad \frac{d^{2} \tilde{\beta}\left(H^{*}+\epsilon \varphi\right)}{d \epsilon^{2}}\right|_{\epsilon=0} \leq 0 .
$$

\section{Lemma 2.}

The constant function $H^{*}=\frac{1}{\gamma}$ is the unique stationary point of the optimization problem (13) over the set $\mathcal{F}_{+}$.

Corollary 2. If $\gamma \geq \frac{1}{H_{0}}$ then the constant function $H^{*}=\frac{1}{\gamma}$ is a local maximum of problem (13). Moreover, it is a global maximum of (13) among all $H$ such that $\sup _{t} H(t) \leq H_{0}$.

Hereafter, the proofs are presented.

1) Proof of Lemma 1: Let $H_{0}$ be the unique solution to the equation $1-2 H_{0}+2 H_{0} \mathrm{e}^{\frac{-1}{H_{0}}}=0$ (see Appendix), and $H \in \mathcal{F}_{+}$.

Since $H \in \mathcal{F}_{+}$and $\varphi$ is bounded, there is $\epsilon_{0}>0$ such that for any $\epsilon$ such that $|\epsilon| \leq \epsilon_{0}$, the constraint in (10) holds. We now explicit the derivatives involved in (15). We have

$$
\begin{aligned}
& \tilde{\beta}(H+\epsilon \varphi)=\int_{0}^{1} \ln \left(1-\mathrm{e}^{\frac{-1}{H(t)+\epsilon \varphi(t)}}\right) d t \\
& \frac{d \tilde{\beta}(H+\epsilon \varphi)}{d \epsilon}=\int_{0}^{1} \frac{\frac{-\varphi(t)}{(H(t)+\epsilon \varphi(t))^{2}} \mathrm{e}^{\frac{-1}{(H(t)+\epsilon \varphi(t))}}}{1-\mathrm{e}^{\frac{-1}{(H(t)+\epsilon \varphi(t))}}} d t \\
& \frac{d^{2} \tilde{\beta}(H+\epsilon \varphi)}{d \epsilon^{2}}=\int_{0}^{1} \frac{d}{d \epsilon}\left(\frac{\frac{-\varphi(t)}{(H(t)+\epsilon \varphi(t))^{2}} \mathrm{e}^{\frac{-1}{(H(t)+\epsilon \varphi(t))}}}{1-\mathrm{e}^{\frac{-1}{(H(t)+\epsilon \varphi(t))}}}\right) d t \\
& =\int_{0}^{1} \frac{\left(\frac{2 \varphi^{2}}{(H-\epsilon \varphi)^{3}}+\frac{\varphi^{2}}{(H+\epsilon \varphi)^{4}}\right) \mathrm{e}^{\frac{-1}{H+\epsilon \varphi}}\left(1-\mathrm{e}^{\frac{-1}{H+\epsilon \varphi}}\right)}{\left(1-\mathrm{e}^{\frac{-1}{H+\epsilon \varphi}}\right)^{2}} d t \\
& +\int_{0}^{1} \frac{\left(\frac{\varphi}{(H+\epsilon \varphi)^{2}} \mathrm{e}^{\frac{-1}{H+\epsilon \varphi}}\right)\left(\frac{-\varphi}{(H+\epsilon \varphi)^{2}} \mathrm{e}^{\frac{-1}{H+\epsilon \varphi}}\right)}{\left(1-\mathrm{e}^{\frac{-1}{H+\epsilon \varphi}}\right)^{2}} d t \\
& \left.\frac{d^{2} \tilde{\beta}(H+\epsilon \varphi)}{d \epsilon^{2}}\right|_{\epsilon=0}=-\int_{0}^{1} \frac{\left(\frac{-2 \varphi^{2}}{H^{3}}+\frac{\varphi^{2}}{H^{4}}\right) \mathrm{e}^{\frac{-1}{H}}\left(1-\mathrm{e}^{\frac{-1}{H}}\right)}{\left(1-\mathrm{e}^{\frac{-1}{H}}\right)^{2}} d t \\
& -\int_{0}^{1} \frac{\frac{\varphi^{2}}{H^{4}} \mathrm{e}^{\frac{-2}{H}}}{\left(1-\mathrm{e}^{\frac{-1}{H}}\right)^{2}} d t \\
& \left.\frac{d^{2} \tilde{\beta}(H+\epsilon \varphi)}{d \epsilon^{2}}\right|_{\epsilon=0}=-\underbrace{\int_{0}^{1} \frac{\frac{\varphi^{2}}{H^{4}} \mathrm{e}^{\frac{-1}{H}}}{\left(1-\mathrm{e}^{\frac{-1}{H}}\right)^{2}}}_{\geq 0} \underbrace{\left(1-2 H+2 H \mathrm{e}^{\frac{-1}{H}}\right)}_{s(H)} d t .
\end{aligned}
$$

In Appendix, we show that $s(H) \geq 0$ whenever $H \leq H_{0}$. We conclude that, if $\sup _{t} H(t) \leq H_{0}$ then

$$
\frac{d^{2} \tilde{\beta}\left(H^{*}+\epsilon \varphi\right)}{d \epsilon^{2}} \leq 0 \text {. }
$$

2) Proof of Lemma 2: Consider $H^{*} \in \mathcal{F}_{+}$. Let $\varphi \in L^{\infty}([0,1])$ be such that (14) holds. We have from (17)

$$
\left.\frac{d \tilde{\beta}(H+\epsilon \varphi)}{d \epsilon}\right|_{\epsilon=0}=\int_{0}^{1} \frac{\frac{-\varphi(t)}{H^{2}(t)} \mathrm{e}^{\frac{-1}{H(t)}}}{1-\mathrm{e}^{\frac{-1}{H(t)}}} d t .
$$

Defining

$$
\zeta(t)=\frac{\mathrm{e}^{\frac{-1}{H^{*}(t)}}}{\left[1-\mathrm{e}^{\frac{-1}{H^{*}(t)}}\right] H^{* 2}(t)},
$$

it follows that (15) is equivalent to,

$$
\int_{0}^{1} \varphi(t) \zeta(t) d t=0
$$

At this stage, we can check that if $H^{*}=\frac{1}{\gamma}$ then $\zeta(t)=c_{0}$ does not depend on $t$, hence we have established that for any $\varphi(t)$ satisfying (14), we must have: $\int_{0}^{1} \zeta(t) \varphi(t) d t=c_{0} \int_{0}^{1} \varphi(t) d t=0$, i.e. (15) holds. This shows, as claimed, that $H^{*}=\frac{1}{\gamma}$ is a stationary point of (13) under the constraints in (10) and (12). We will now prove the converse.

We assume now that $H^{*} \in F_{+}$is a stationary point of (13) with the constraints in (10) and (12). What we have just established is that (20) must hold for all $\varphi$ that satisfies (14). $\zeta$ is then orthogonal to all the zero mean functions $\varphi \in L^{\infty}$. Thus, $\zeta$ is a constant $c_{0}$, i.e.

$$
\frac{\mathrm{e}^{\frac{-1}{H^{*}(t)}}}{\left[1-\mathrm{e}^{\frac{-1}{H^{*}(t)}}\right] H^{*^{2}}(t)}=c_{0} .
$$

Hence, $\exists c_{0} \in \mathbb{R}$ such that $\forall t \in[0,1] H^{*}(t)$ belongs to the set of solutions of the equation $J(H)=c_{0}$ with

$$
J(H)=\frac{\mathrm{e}^{\frac{-1}{H}}}{\left[1-\mathrm{e}^{\frac{-1}{H}}\right] H^{2}} .
$$

To conclude that $H^{*}$ itself be constant, we now analyse the variations of the function $J(H)$.

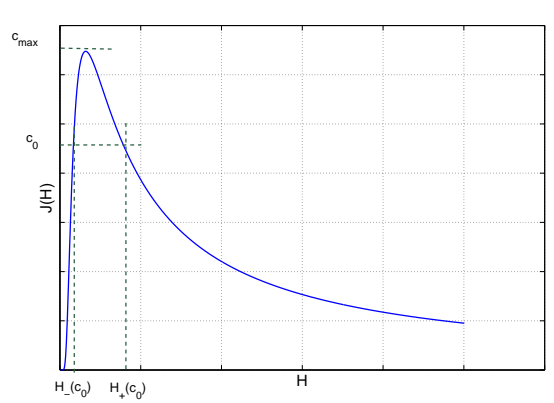

Figure 1: Curve of the function $J(H)$.

The simulation of $J(H)$ in Fig. 1, shows that for a certain value $c_{0}$, the line of equation $J(H)=c_{0}$ cuts the curve of the function $J$ in a single point which coincides with the maximum value of $J$ that we note $c_{\max }$, and two distinct points when $c_{0}$ is less than $c_{\max }$. When $c_{0}$ is greater than $c_{\max }$ the line does not cut the curve of $J$.

Thus, the set $S_{J}$ of solutions for (21) is

$$
S_{J}= \begin{cases}H_{+}\left(c_{0}\right), H_{-}\left(c_{0}\right) & \text { if } 0<c_{0} \leq c_{\max } \\ \emptyset & \text { if } c_{0}>c_{\max }\end{cases}
$$


Table I: Solutions of (21) for different values of $c_{0}$.

\begin{tabular}{|l|c|c|}
\hline$c_{0}$ & $H_{-}\left(c_{0}\right)$ & $H_{+}\left(c_{0}\right)$ \\
\hline 0.64 & 0.720 & 00.72 \\
0.62 & 0.510 & 00.83 \\
0.60 & 0.450 & 00.90 \\
0.50 & 0.360 & 01.34 \\
0.40 & 0.300 & 01.90 \\
0.30 & 0.250 & 02.76 \\
0.20 & 0.210 & 04.46 \\
0.10 & 0.170 & 09.48 \\
0.05 & 0.145 & 19.50 \\
0.02 & 0.091 & 49.49 \\
0.01 & 0.085 & 99.49 \\
\hline
\end{tabular}

Note that when $c_{0}=c_{\max }$, we have $H_{+}\left(c_{0}\right)=H_{-}\left(c_{0}\right)$. $H_{+}\left(c_{0}\right)$ is the greatest solution, and $H_{-}\left(c_{0}\right)$ is the smallest one $\left(H_{-}\left(c_{0}\right) \leq H_{+}\left(c_{0}\right)\right)$.

The following property summarizes what we have established so far

Property 2. Let $H^{*} \in \mathcal{F}_{+}$be a stationary point of $\tilde{\beta}$, under the constraints in (10) and (12). There exists a constant $c_{0} \in\left[0, c_{\max }\right]$, a set $\mathcal{A}_{+}$and a set $\mathcal{A}_{-}=[0,1] \backslash \mathcal{A}_{+}$such that

$$
H_{\mid \mathcal{A}_{+}}=H_{+}\left(c_{0}\right), \text { and } H_{\mid \mathcal{A}_{-}}=H_{-}\left(c_{0}\right) .
$$

$H_{\mid \mathcal{A}_{+}}\left(H_{\mid \mathcal{A}_{-}}\right.$respectively) is the restriction of the function $H$ over the set $\mathcal{A}_{+} \subset[0,1]\left(\mathcal{A}_{-} \subset[0,1]\right.$ respectively).

Corollary 3. The Lebesgue measure of the interval $\mathcal{A}_{+}$can be expressed as

$$
\tilde{L}_{\mathcal{A}_{+}\left(c_{0}\right)}=\frac{\frac{1}{\gamma}-H_{-}\left(c_{0}\right)}{H_{+}\left(c_{0}\right)-H_{-}\left(c_{0}\right)} \in[0,1] .
$$

In fact, from (12) and Property 1, we have

$$
\begin{gathered}
\tilde{L}_{\mathcal{A}_{+}}\left(c_{0}\right) H_{+}\left(c_{0}\right)+\left(1-\tilde{L}_{\mathcal{A}_{+}}\right) H_{-}\left(c_{0}\right)=\frac{1}{\gamma}, \\
\tilde{L}_{\mathcal{A}_{+}}\left(c_{0}\right)\left(H_{+}\left(c_{0}\right)-H_{-}\left(c_{0}\right)\right)=\frac{1}{\gamma}-H_{-}\left(c_{0}\right) .
\end{gathered}
$$

Property 3. Let $H^{*} \in \mathcal{F}_{+}$be a stationary point of $\tilde{\beta}$, under the constraints in (10) and (12). Then, the value of $c_{0}$ solves the following optimization problem

$$
\begin{aligned}
\underset{c_{0}}{\operatorname{maximize}} \quad \tilde{\beta}\left(c_{0}\right) & =\tilde{L}_{\mathcal{A}_{+}}\left(c_{0}\right) \ln \left(1-\mathrm{e}^{-\frac{1}{H_{+}\left(c_{0}\right)}}\right) \\
& +\left(1-\tilde{L}_{\mathcal{A}_{+}}\left(c_{0}\right)\right) \ln \left(1-\mathrm{e}^{-\frac{1}{H_{-}\left(c_{0}\right)}}\right),
\end{aligned}
$$

subject to $\quad \tilde{L}_{\mathcal{A}_{+}}\left(c_{0}\right) \in[0,1]$.

Numerical Results: Table I shows for each value of $c_{0}$ the set of solutions $S_{J}$ of (21). As we can see, $H_{-}\left(c_{0}\right)$ is an increasing function of $c_{0}$ and $H_{+}\left(c_{0}\right)$ is a decreasing function of $c_{0}$, we can resume these conclusions in Table II.

Now, we should study the variations of $\tilde{\beta}\left(c_{0}\right)$, which depend on the monotonicity of $\tilde{L}_{\mathcal{A}^{+}}$. We have $\frac{1}{\gamma} \geq H_{-}$ since $\tilde{L}_{\mathcal{A}^{+}}$is positive, so we cannot decide directly on the monotonicity of $\tilde{L}_{\mathcal{A}^{+}}$, because it is the product of a positive decreasing function $c_{0} \mapsto \frac{1}{\gamma}-H_{-}\left(c_{0}\right)$ and a positive increasing function $c_{0} \mapsto \frac{1}{H_{+}\left(c_{0}\right)-H_{-}\left(c_{0}\right)}$. Therefore, we simulate the variations of $\tilde{L}_{\mathcal{A}^{+}}$and $\tilde{\beta}\left(c_{0}\right)$ as depicted in Fig. 2 and Fig. 3.

To maximize $\tilde{\beta}$ we should minimize $\tilde{L}_{\mathcal{A}^{+}}$under the constraint
Table II: Variations of $H_{+}, H_{-}$and $\frac{1}{H_{+}-H_{-}}$as a function of $c_{0}$.

\begin{tabular}{|c|cc|}
\hline$c_{0}$ & 0 & $c_{\max }$ \\
\hline$H_{+}$ & $+\infty \longrightarrow H_{\max }$ \\
\hline$H_{-}$ & $0 \longrightarrow+H_{\max }$ \\
\hline$\frac{1}{H_{+}-H_{-}}$ & $0 \longrightarrow+\infty$ \\
\hline
\end{tabular}

of $0 \leq \tilde{L}_{\mathcal{A}^{+}} \leq 1$. For $\tilde{L}_{\mathcal{A}^{+}}=0$, we have $H_{-}=\frac{1}{\gamma}$ and $\tilde{\beta}^{*}=\ln \left(1-\mathrm{e}^{-\gamma}\right)$. Thus, $H^{*}$ takes a single value $H_{-}$and $H^{*}=\frac{1}{\gamma}$. To conclude, for $H^{*} \in \mathcal{F}_{+}$a stationary point of $\tilde{\beta}$ under the constraint in (12), we have $H^{*}=\frac{1}{\gamma}$. This concludes the proof of Lemma 2.

3) Proof of Corollary 2: The set $\mathcal{F}_{+} \cap\left\{H: \sup _{t} H(t) \leq\right.$ $\left.H_{0}\right\}$ is convex. We deduce from Lemma 1 that $\tilde{\beta}$ is a concave function on this convex set. Therefore, its local maximum is a global maximum over this set [32]. From Lemma 2, $H^{*}=\frac{1}{\gamma}$ is the unique stationary point of $\tilde{\beta}$ over $\mathcal{F}_{+}$, hence it is the global maximum of $\tilde{\beta}$ over $\mathcal{F}_{+} \cap\left\{H: \sup _{t} H(t) \leq H_{0}\right\}$.

4) Proof of the main result: Property 1: Property 1 follows from Corollary 2 using two simple observations that we highlight:

- The Property (4) is equivalent to the condition $H(t)=H^{*}(t)=\frac{P}{\gamma T}$;

- The property $\gamma \geq \gamma_{\text {crit }}\left(\left\{g_{m}\right\}\right)$ is equivalent to the condition $\sup _{t} H(t) \leq H_{0}$.

\section{ANALYSis By Simulations}

In order to illustrate our theoretical results, we consider three different multicarrier modulation systems, which are based on different families of modulation functions, and we analysed the CCDF of their PAPR. A comparison in terms of PAPR performance, between each considered multicarrier system and the conventional OFDM is presented.

\section{A. Walsh-Hadamard-MC (WH-MC)}

Instead of using the IFFT for the modulation, we can use the inverse Walsh-Hadamard transform (IWHT). The family of the modulation functions is expressed as:

$$
g_{m}[k]=W_{q}[k],
$$

where $W_{q}$ represent the Walsh functions (see Fig. 4) and are columns of Hadamard matrix of dimension $M=2^{Q}$, which is defined by the following recursive formula:

$$
H_{w}\left(2^{1}\right)=\left(\begin{array}{cc}
1 & 1 \\
1 & -1
\end{array}\right)
$$

and for $2 \leq q \leq Q$ :

$H_{w}\left(2^{q}\right)=\left(\begin{array}{cc}H_{w}\left(2^{q-1}\right) & H_{w}\left(2^{q-1}\right) \\ H_{w}\left(2^{q-1}\right) & -H_{w}\left(2^{q-1}\right)\end{array}\right)=H_{w}(2) \otimes H_{w}\left(2^{q-1}\right)$, 


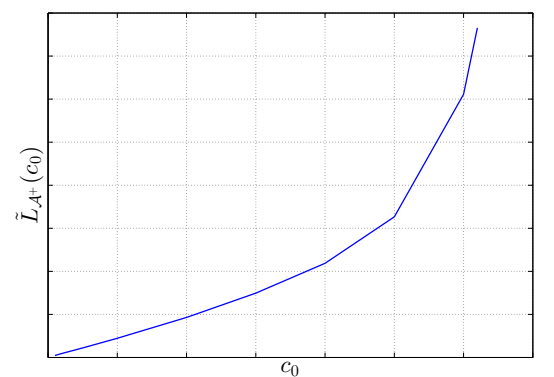

Figure 2: Curve of the function $\tilde{L}_{\mathcal{A}^{+}}\left(c_{0}\right)$.

where $\otimes$ denotes the Kronecker product.

Note that the Hadamard matrix consists only of +1 and -1 entries, that is why the implementation has a simple structure featuring only additions and subtractions. In fact, IWHT can be implemented using the radix-2 algorithm, which means that there are only $M \log _{2} M$ required complex additions [33].

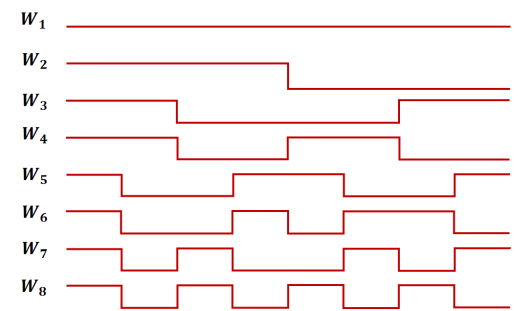

Figure 4: Walsh-Hadamard functions.

Fig. 4 depicts the shape of the Walsh functions for $Q=3$. As we can notice, all the functions have the same modulus and this modulus is constant over time. From Corollary 1, WH-MC has the same PAPR performance as conventional OFDM.

Let us check this conclusion by simulation. To do so, we generate 10000 realizations of the WH-MC symbol using the quadrature phase-shift keying (QPSK) constellation, and $M=$ 64 carriers. The CCDF of the PAPR of WH-MC and OFDM are compared in Fig. 5.

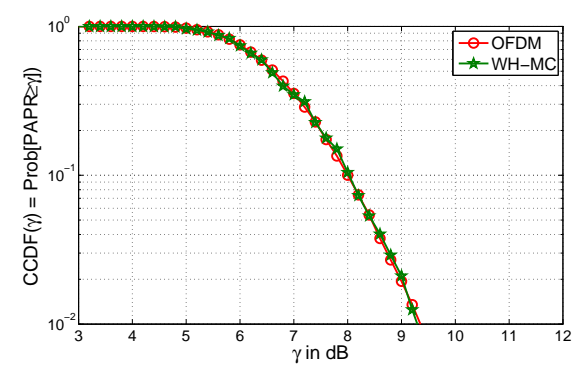

Figure 5: CCDF of the PAPR for conventional OFDM and WH-MC.

We can observe that OFDM and WH-MC have the same PAPR distribution function, hence the same PAPR performance. Indeed, this observation is consistent with the theoretical predictions undertaken in Corollary 1 .

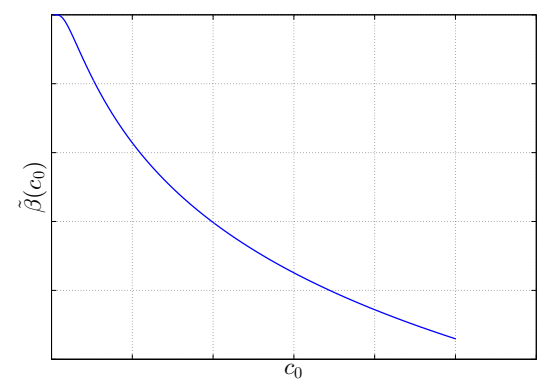

Figure 3: Curve of the function $\tilde{\beta}\left(c_{0}\right)$.

\section{B. WCP-OFDM}

Weighted cyclic prefix-OFDM (WCP-OFDM) is another variant of OFDM, which gives a weighted version of the cyclic prefix-OFDM, by using non-rectangular pulse shapes. The prototype filter out-of-band energy (OBE) defined in [34] is used in WCP-OFDM. In this case, the family of modulation functions is expressed as $g_{m}[k]=g[k] e^{j 2 \pi \frac{m}{M} k}$ such that $g[k]$ is defined as 5

$$
g[k] \mathrm{e}^{j 2 \pi \frac{m}{M} k}=\left\{\begin{array}{l}
\frac{1}{\sqrt{M^{\prime}}} \cos \left(a+b \frac{2 k+1}{2 \Delta}\right) \mathrm{e}^{j 2 \pi \frac{m}{M}[k]} \\
\text { if } 0 \leq k \leq \Delta-1, \\
\frac{1}{\sqrt{M^{\prime}}} \mathrm{e}^{j 2 \pi \frac{m}{M}[k]} \\
\text { if } \Delta \leq k \leq M^{\prime}-1, \\
\frac{1}{\sqrt{M^{\prime}}} \cos \left(a+b \frac{2(M-k)+1}{2 \Delta}\right) \mathrm{e}^{j 2 \pi \frac{m}{M}[k]} \\
\text { if } \quad M^{\prime} \leq k \leq M-1, \\
0, \quad \text { else. }
\end{array}\right.
$$

with $g[k]$ is the OBE filter.

We can easily check that $g[k]$ satisfies the condition in (2). In addition, we notice that $\forall m \in \llbracket 0, M-1 \rrbracket$ the modulus $\left|g_{m}[k]\right|^{2}=\left|g[k] \mathrm{e}^{j 2 \pi \frac{m}{M} k}\right|^{2}=|g[k]|^{2}$ depends on time. From Corollary 1 , the PAPR performance of WCP-OFDM has to be worse than conventional OFDM system.

To approve this conclusion, we simulate the CCDF of the PAPR by considering the OBE filter.

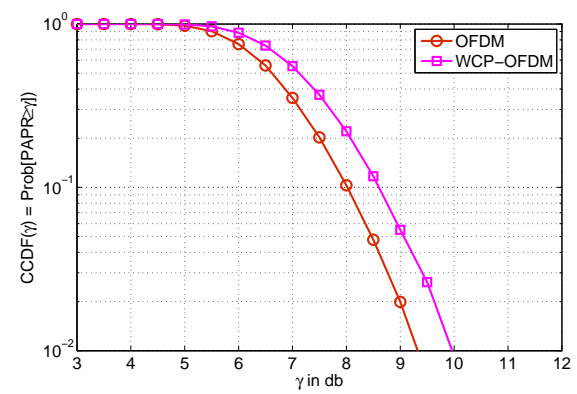

Figure 6: CCDF of the PAPR for conventional OFDM and WCP-OFDM.

Fig. 6 represents a comparison of the CCDF of the PAPR between conventional OFDM and WCP-OFDM. We can notice that the curve of WCP-OFDM is shifted to the right, compared

\footnotetext{
${ }^{5} M=80, M^{\prime}=4 / 5 M, \Delta=M^{\prime}-M, M_{0}=\frac{M}{\Delta}, b=\frac{1}{\alpha+\beta M_{0}}, a=$ $\frac{\pi}{4}-\frac{1}{2} b, \alpha=-0.1714430594740783, \beta=-0.5852184808129936$.
} 
to OFDM. Thus, OFDM has a better PAPR performance than WCP-OFDM, which matches our theoretical results.

\section{Wavelet-OFDM}

Wavelet-OFDM, or also known as orthogonal wavelet division multiplexing (OWDM) [35], is a multicarrier system based on the wavelet transform. The principle of the wavelet transform is to decompose the signal in terms of small waves components called wavelets. The Wavelet-OFDM transmitted signal can be defined as:

$$
\begin{aligned}
X(t)= & \sum_{n} \sum_{j=J_{0}}^{J-1} \sum_{k=0}^{2^{j}-1} w_{j, k} \psi_{j, k}(t-n T) \\
& +\sum_{n} \sum_{q=0}^{2^{J_{0}-1}} a_{J_{0}, q} \phi_{J_{0}, q}(t-n T) .
\end{aligned}
$$

- $J-1$ : last scale considered, with $M=2^{J}$;

- $J_{0}$ : first scale considered $\left(J_{0} \leq j \leq J-1\right)$;

- $w_{j, k}$ : wavelet coefficients located at $k$-th position from scale $j$;

- $a_{J_{0}, q}$ : approximation coefficients located at $q$-th position from the first scale $J_{0}$;

- $\psi_{j, k}=2^{j / 2} \psi\left(2^{j} t-k T\right)$ : the wavelet orthonormal family, $\psi$ is the mother wavelet function;

- $\phi_{J_{0}, q}=2^{\frac{J_{0}}{2}} \phi\left(2^{J_{0}} t-q T\right)$ : the scaling orthonormal family at the scale $J_{0}, \phi$ is the mother scaling function.

Note that the wavelet functions and the scaling functions have identical energy. For more details about the wavelet theory, the reader can refer to [36].

Several wavelets can be used to modulate the input symbols, such as Daubechies, Coiflets, and Symlets. We are interested here to the Haar wavelet, which belongs to the family of Daubechies wavelets. The Haar mother wavelet function $\psi_{\text {haar }}(t)$ is expressed as:

$$
\text { with } \quad \psi_{\text {haar }}(t)= \begin{cases}\frac{1}{\sqrt{T}}, & \text { if } 0 \leq t \leq \frac{T}{2} \\ -\frac{1}{\sqrt{T}}, & \text { if } \frac{T}{2} \leq t \leq T \\ 0, & \text { else }\end{cases}
$$

The scaling function $\phi_{\text {haar }}(t)$ can be described as:

$$
\text { and } \quad \phi_{\text {haar }}(t)= \begin{cases}\frac{1}{\sqrt{T}} & \text { if } 0 \leq t \leq T, \\ 0, & \text { else. }\end{cases}
$$

Fig. 7 describes Haar wavelet functions $\psi_{j, k}^{\text {haar }}$ for $J_{0}=0$

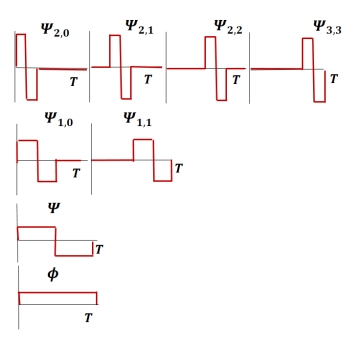

Figure 7: Haar wavelet function for different scales.

and $M=8$. As we can notice, the temporal support of the contracted versions of the mother wavelet function $\psi^{\text {haar }}$ are smaller than the symbol period $T$, therefore this family of functions does not satisfy the constraint in (2). From Property III-A, we can reach a better PAPR performance than using the Fourier transform. To check this claim by simulation, we consider the Haar wavelet transform, and we extract the detail and approximation coefficients at the maximal level 6 $\left(J_{0}=0\right)$ for a number of carriers $M=64$.

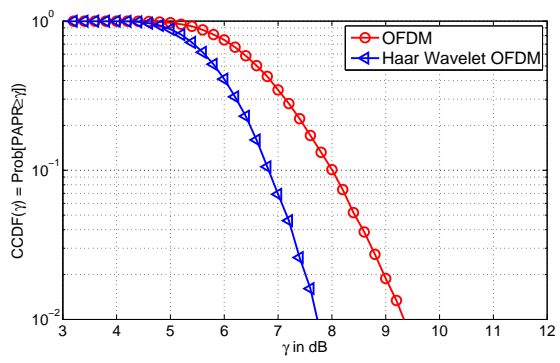

Figure 8: CCDF of the PAPR for conventional OFDM and Haar Wavelet based OFDM.

We can observe in Fig. 8 that the curve of the Haar WaveleOFDM is shifted to the left, compared to OFDM. Thus, the Haar Wavelet-OFDM has a better PAPR performance than conventional OFDM.

Note here that, by changing the modulation waveform, the PAPR is reduced. As explained previously, without using the classical PAPR reduction methods, the PAPR can be reduced by selecting the appropriate waveform.

Complexity of implementation of Wavelet-OFDM: according to the Mallat Algorithm in [36], the wavelet modulation can be implemented based on the inverse discrete wavelet transform (IDWT). The IDWT consists of up-sampling by a factor of two and filtering the approximation coefficients $a_{j, k}$ (scaling coefficients) and the detail coefficients $w_{j, k}$ (wavelet coefficients) respectively by a low-pass filter $g$ and a high-pass filter $h$ associated to the wavelet used. Let $L$ be the length of the filters $h$ and $g$ ( $L=2$ in the case of the Haar wavelet). Let $M=2^{J}$ be the number of carriers which is equal to the number of the input coefficients. The wavelet modulation is calculated with a number of operations bounded by

$$
\sum_{j=J_{0}+1}^{J} 2^{j} L \leq \sum_{j=1}^{J} 2^{j} L
$$

The complexity order in terms of the number of additions and multiplications is therefore $\mathcal{O}(M L)$. Knowing that the complexity order of the FFT or the IFFT is $\mathcal{O}\left(M \log _{2}(M)\right)$, the complexity increase order is about $\mathcal{O}\left(\frac{L}{\log _{2}(M)}\right)$, which is affordable since $L$ is bounded, and the number of carriers $M$ is usually large.

Other examples of multicarrier systems from the literature can also be discussed:

- The PAPR of NOFDM is evaluated by simulation for Hanning and Kaiser windows in [12]. The PAPR performance of NOFDM is shown experimentally to be worse than OFDM. Corollary 1 gives a theoretical explanation to these simulation results. 
- According to the necessary condition theorem, we understand now why FBMC systems based on IOTA or SRRC filters for example as well as the universal-filtered multicarrier (UFMC) systems [37] do not have better PAPR than OFDM, since they do not satisfy the necessary condition stated in the theorem.

Fig. 9 summarizes the conclusions of this study. The rectangle represents the set of all GWMC waveforms $\in L_{I}^{\infty}$. The optimization problem analysed in this work is for the waveforms belonging to the set $A$, which means satisfying (2). Systems in $A \cap B$ (including OFDM, WH-MC) have the best possible PAPR performance among all systems in A. Any system with better PAPR performance than OFDM must be in $C$. There are indeed systems (Daubechies 6, Symlet 3, Coiflet 2) in $C$ with better PAPR performance than OFDM. Some are even in $B$ (Haar wavelets), but not in $A$.

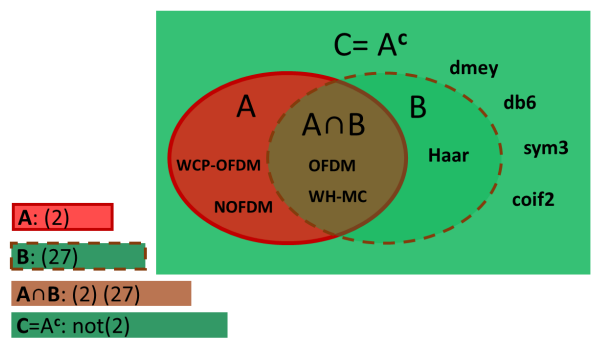

Figure 9: Taxonomy of multicarrier waveforms regarding the PAPR performance.

\section{CONCLUSiON}

In this paper, we have investigated the GWMC system based on the family of modulation functions (the modulation transform and the pulse shaping filter) which does not vanish in the symbol period, and we have proved analytically that the PAPR, which depends on the modulation waveform, is optimal only if the sum of these waveforms over the number of carriers and the number of symbols is constant over time. We have concluded that there exists an infinite number of GWMC systems that are optimal in terms of PAPR performance, and conventional OFDM based on the Fourier transform and the rectangular filter belongs to this family. In addition, we have deduced that the PAPR performance of GWMC systems cannot be better than OFDM system without reducing the temporal support of the modulation functions compared to the symbol period. It is worth mentioning that, by limiting the support of the individual carriers, we are moving more and more from a multicarrier system to a single carrier system.

We have provided some examples to illustrate our theoretical results: WH-MC has a PAPR performance equal to that of OFDM, which is optimal among systems which satisfy condition (2), the WCP-OFDM's waveform is not constant over time and thus it is worse than conventional OFDM in terms of PAPR performance. By not satisfying constraint (2), it is possible to achieve a better PAPR performance, as has been shown for the Haar wavelets. Moreover, our work also provides a theoretical explanation about why several advanced
Table III: Study of the positivity of the function $s$.

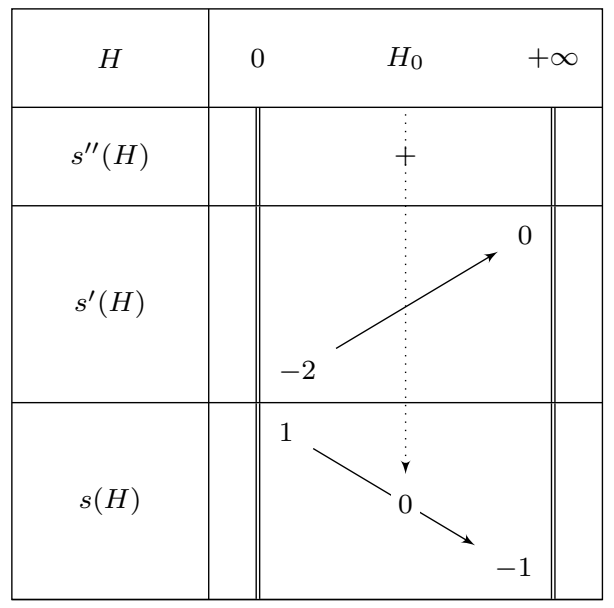

multicarrier systems such as NOFDM, FBMC, UFMC, do not achieve a better PAPR than OFDM.

Our future work will focus on designing a new waveform with low PAPR than OFDM, by acting on the number of intervals that vanish over time, and taking into consideration other constraints such as the bit error rate, the complexity and the spectral efficiency. If we would like to design a new waveform as a candidate for the next generation of mobile communication systems, it should not be selected from the excluded region $A$, because in this case, it will not improve the PAPR performance. Waveforms with reduced PAPR can be found in particular in the region $B$ (as depicted in Fig. 9).

\section{APPENDIX}

We study the variations of the function $s(H)=1-2 H+2 H \mathrm{e}^{\frac{-1}{H}}$, we have

$$
\begin{aligned}
s^{\prime}(H) & =-2+2 \mathrm{e}^{\frac{-1}{H}}+\frac{2}{H} \mathrm{e}^{\frac{-1}{H}}, \\
s^{\prime \prime}(H) & =\frac{2}{H^{2}} \mathrm{e}^{\frac{-1}{H}}+2\left(-\frac{1}{H^{2}} \mathrm{e}^{\frac{-1}{H}}+\frac{1}{H} \frac{1}{H^{2}} \mathrm{e}^{\frac{-1}{H}}\right), \\
& =\frac{2}{H^{3}} \mathrm{e}^{\frac{-1}{H}} \geq 0 .
\end{aligned}
$$

As we can see in Table III, the function $s$ is positive when $0<H \leq H_{0}$. A numerical approximation gives $H_{0} \approx 0.63$.

\section{REFERENCES}

[1] H. Ochiai and H. Imai, "On the Distribution of the Peak-to-Average Power Ratio in OFDM Signals," Communications, IEEE Transactions on, vol. 49, no. 2, pp. 282-289, 2001.

[2] T. Jiang, M. Guizani, H.-H. Chen, W. Xiang, and Y. Wu, "Derivation of PAPR Distribution for OFDM Wireless Systems Based on Extreme Value Theory," Wireless Communications, IEEE Transactions on, vol. 7, no. 4, pp. 1298-1305, 2008.

[3] G. Auer, O. Blume, V. Giannini, I. Godor, M. Imran, Y. Jading, E. Katranaras, M. Olsson, D. Sabella, P. Skillermark et al., "D2. 3: Energy Efficiency Analysis of the Reference Systems, Areas of Improvements and Target Breakdown," EARTH, 2010. 
[4] R. V. Nee and R. Prasad, OFDM for Wireless Multimedia Communications. Inc.: Artech House, 2000.

[5] A. Skrzypczak, P. Siohan, and J.-P. Javaudin, "Analysis of the Peakto-Average Power Ratio for OFDM/OQAM," in Signal Processing Advances in Wireless Communications, 2006. SPAWC'06. IEEE 7th Workshop on. IEEE, 2006, pp. 1-5.

[6] M. Chafii, J. Palicot, and R. Gribonval, "Closed-form Approximations of the Peak-to-Average Power Ratio Distribution for Multi-Carrier Modulation and their Applications," EURASIP Journal on Advances in Signal Processing, vol. 2014, no. 1, pp. 1-13, 2014.

[7] T. Jiang and G. Zhu, "Complement block coding for reduction in peakto-average power ratio of ofdm signals," Communications Magazine, IEEE, vol. 43, no. 9, pp. S17-S22, 2005.

[8] M. Deumal, A. Behravan, and J. L. Pijoan, "On Cubic Metric Reduction in OFDM Systems by Tone Reservation," Communications, IEEE Transactions on, vol. 59, no. 6, pp. 1612-1620, 2011.

[9] C. Li, T. Jiang, Y. Zhou, and H. Li, "A Novel Constellation Reshaping Method for PAPR Reduction of OFDM Signals," Signal Processing, IEEE Transactions on, vol. 59, no. 6, pp. 2710-2719, 2011.

[10] T. Jiang and C. Li, "Simple Alternative Multisequences for PAPR Reduction without Side Information in SFBC MIMO-OFDM Systems," Vehicular Technology, IEEE Transactions on, vol. 61, no. 7, pp. 33113315, 2012.

[11] S. Y. Le Goff, B. K. Khoo, C. C. Tsimenidis, and B. S. Sharif, "A Novel Selected Mapping Technique for PAPR Reduction in OFDM Systems," Communications, IEEE Transactions on, vol. 56, no. 11, pp. 1775-1779, 2008.

[12] H. Bogucka, A. M. Wyglinski, S. Pagadarai, and A. Kliks, "Spectrally Agile Multicarrier Waveforms for Opportunistic Wireless Access," Communications Magazine, IEEE, vol. 49, no. 6, pp. 108-115, 2011.

[13] A. Sahin, I. Guvenc, and H. Arslan, "A Survey on Multicarrier Communications: Prototype Filters, Lattice Structures, and Implementation Aspects," Communications Surveys \& Tutorials, IEEE, vol. 16, no. 3, pp. 1312-1338, 2012.

[14] W. Kozek and A. F. Molisch, "Nonorthogonal Pulseshapes for Multicarrier Communications in Doubly Dispersive Channels," Selected Areas in Communications, IEEE Journal on, vol. 16, no. 8, pp. 1579-1589, 1998.

[15] P. Siohan, C. Siclet, and N. Lacaille, "Analysis and Design of OFDM/OQAM Systems based on Filterbank Theory," Signal Processing, IEEE Transactions on, vol. 50, no. 5, pp. 1170-1183, 2002.

[16] L. Vangelista and N. Laurenti, "Efficient Implementations and Alternative Architectures for OFDM-OQAM Systems," Communications, IEEE Transactions on, vol. 49, no. 4, pp. 664-675, 2001.

[17] T. Jiang, C. Ni, D. Qu, and C. Wang, "Energy-efficient NCOFDM/OQAM-based Cognitive Radio Networks," Communications Magazine, IEEE, vol. 52, no. 7, pp. 54-60, 2014.

[18] B. Farhang-Boroujeny, "OFDM versus Filter Bank Multicarrier," Signal Processing Magazine, IEEE, vol. 28, no. 3, pp. 92-112, 2011.

[19] B. Le Floch, M. Alard, and C. Berrou, "Coded Orthogonal Frequency Division Multiplex," Proceedings of the IEEE, vol. 83, no. 6, pp. 24$182,1995$.

[20] G. Fettweis, M. Krondorf, and S. Bittner, "Gfdm-generalized frequency division multiplexing," in Vehicular Technology Conference, 2009. VTC Spring 2009. IEEE 69th. IEEE, 2009, pp. 1-4.

[21] N. Michailow, I. Gaspar, S. Krone, M. Lentmaier, and G. Fettweis, "Generalized frequency division multiplexing: Analysis of an alternative multi-carrier technique for next generation cellular systems," in Wireless
Communication Systems (ISWCS), 2012 International Symposium on. IEEE, 2012, pp. 171-175.

[22] R. Kanti and M. Rai, "Comparative Analysis of Different Wavelets in OWDM with OFDM for DVB-T," International Journal of Advancements in Research \& Technology, vol. 2, no. 3, 2013.

[23] K. M. Wong, J. Wu, T. N. Davidson, Q. Jin, and P.-C. Ching, "Performance of Wavelet Packet-Division Multiplexing in Impulsive and Gaussian Noise," Communications, IEEE Transactions on, vol. 48, no. 7, pp. 1083-1086, 2000.

[24] A. Aboltins, "Comparison of Orthogonal Transforms for OFDM Communication System," Electronics and Electrical Engineering Journal, vol. 111, no. 5, pp. 77-80, 2011.

[25] M. Bernhard and J. Speidel, "Multicarrier Transmission using Hadamard Transform for Optical Communications," ITG-Fachbericht-Photonische Netze, 2013.

[26] P. Tan and N. C. Beaulieu, "A Comparison of DCT-based OFDM and DFT-based OFDM in Frequency Offset and Fading Channels," Communications, IEEE Transactions on, vol. 54, no. 11, pp. 2113-2125, 2006.

[27] G. D. Mandyam, "On the Discrete Cosine Transform and OFDM Systems," in Acoustics, Speech, and Signal Processing, 2003. Proceedings.(ICASSP'03). 2003 IEEE International Conference on, vol. 4. IEEE, 2003, pp. IV-544.

[28] A. Deshmukh and S. Bodhe, "Performance of DCT Based OFDM Communication System Working in $60 \mathrm{GHz}$ Band," International Journal of Engineering Science \& Technology, vol. 4, no. 1, 2012.

[29] N. De Bruijn, "Uncertainty Principles in Fourier Analysis," Inequalities, vol. 2, pp. 57-71, 1967.

[30] A. Skrzypczak, P. Siohan, and J.-P. Javaudin, "Peak-to-Average Power Ratio Issues for Pulse-Shaped Multicarrier Modulations," in Advances on Processing for Multiple Carrier Schemes: OFDM \& OFDMA, F. Bader and N. Zorba, Eds. Nova Science Publishers, Inc., 2011, pp. 43-90.

[31] A. Kliks, "New Transmission and Reception Techniques of the Generalized Multicarrier Signals," Ph.D. dissertation, Poznan University of Technology, 2011.

[32] S. Boyd and L. Vandenberghe, Convex Optimization. Cambridge University Press, 2004.

[33] B. Evans et al., "Hardware Structure for Walsh-Hadamard Transforms," Electronics Letters, vol. 34, no. 21, pp. 2005-2006, 1998.

[34] D. Roque, "Modulations Multiporteuses WCP-OFDM: évaluation des performances en environnement radiomobile," Ph.D. dissertation, Grenoble University, 2012.

[35] S. L. Linfoot, M. K. Ibrahim, and M. M. Al-Akaidi, "Orthogonal Wavelet Division Multiplex: An Alternative to OFDM," Consumer Electronics, IEEE Transactions on, vol. 53, no. 2, pp. 278-284, 2007.

[36] S. Mallat, A Wavelet Tour of Signal Processing. Academic press, 1999.

[37] F. Schaich and T. Wild, "Waveform Contenders for 5G-OFDM vs. FBMC vs. UFMC," in Communications, Control and Signal Processing (ISCCSP), 2014 6th International Symposium on. IEEE, 2014, pp. $457-460$. 


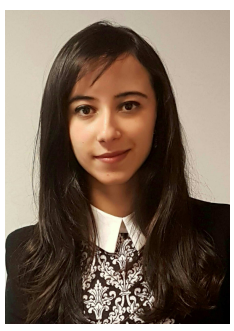

Marwa Chafii (M'13) is currently doing her $\mathrm{PhD}$ at the Communication and Electronic Embedded Systems (SCEE) research team of CentraleSupélec, Campus of Rennes-France. Her research interests include advanced waveforms for multi-carrier systems and the peak-to-average power ratio (PAPR) reduction problem. She received in 2013, her research master's degree in the field of advanced wireless communication systems (SAR) from CentraleSupélec at Paris-France, and her engineering diploma in telecommunications from Institut $\mathrm{Na}-$ tional des Postes et Télécommunications (INPT) in Morocco. Between 2014 and 2015, she has been visiting, for short scientific research missions, Poznan University of Technology (Poland), University of York (UK), and Yokohama National University (Japan).

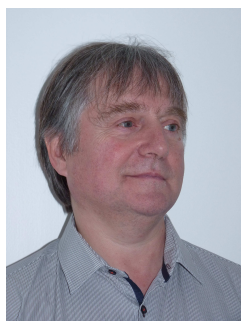

Jacques Palicot received, in 1983, his $\mathrm{PhD}$ degree in Signal Processing from the University of Rennes. Since 1988, he has been involved in studies about equalization techniques applied to digital transmissions and analog TV systems. Since 1991 he has been involved mainly in studies concerning the digital communications area and automatic measurements techniques. He has taken an active part in various international bodies EBU, CCIR, URSI, and within RACE, ACTS and IST European projects. He has published various scientific articles notably on equalization techniques, echo cancellation, hierarchical modulations and Software Radio techniques. He is author or co-author of more than 300 publications with more than 50 in journals, two books and 22 patents. He is currently involved in adaptive Signal Processing, digital communications, Software Radio, Cognitive radio and Green Radio. From November 2001 to September 2003 he had a temporary position with INRIA/IRISA in Rennes. He serves as Associate Editor for EURASIP JASP since 2008. He also served as lead guest editor for several Special Issues on Software Radio, Cognitive Radio and Green Radio. He was Co General Chairman of ISCIT 2011, Co General Chairman of Next-GWiN 2014, Technical Program Chairman of CROWNCOM 2009, Technical Program Chairman of GREENCOM 2013 and Technical Program Chairman of CRN Symposium of ICC 2014. Since October 2003 he is with CentraleSup $\tilde{A} \odot$ lec in Rennes where he leads the Signal Communications and Embedded Electronics (SCEE) research team.

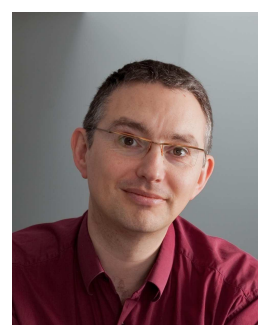

Rémi Gribonval (M'02,SM'08,FM'14) is a Senior Researcher with Inria (Rennes, France), and the scientific leader of the PANAMA research group on sparse audio processing. A former student at École Normale Supérieure (Paris, France), he received the $\mathrm{Ph}$. D. degree in applied mathematics from Université de Paris-IX Dauphine (Paris, France) in 1999, and his Habilitation à Diriger des Recherches in applied mathematics from Université de Rennes I (Rennes, France) in 2007. His research focuses on mathematical signal processing, machine learning, approximation theory and statistics, with an emphasis on sparse approximation, audio source separation and compressed sensing. He founded the series of international workshops SPARS on Signal Processing with Adaptive/Sparse Representations. In 2011, he was awarded the Blaise Pascal Award in Applied Mathematics and Scientific Engineering from the SMAI by the French National Academy of Sciences, and a starting investigator grant from the European Research Council.

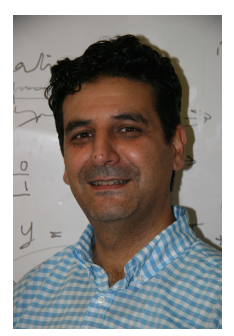

Faouzi Bader (SM'07) received his $\mathrm{PhD}$ degree (with Honours) in Telecommunications in 2002 from Universidad Politécnica de Madrid (UPM), MadridSpain. He joined the Centre Technologic de Telecomunicacions de Catalunya (CTTC) in BarcelonaSpain as Associate researcher in 2002, and nominated in 2006 Senior Research Associate at same institution. Since June 2013, he is as Associate Professor at CentraleSupélec in Rennes- France. His research activities mainly focus on advanced multicarrier waveforms (OFDM(A), (non-) uniform multimode filter based multicarrier schemes) and frequency allocation techniques in relay cognitive environment. He has been involved in several European projects from the 5th-7th EC research frameworks, and from 2012-2013 he was nominated general coordinator and manager of the EC funded research project ICT "EMPhAtiC" focusing on "Enhanced Multicarrier Techniques for Professional Ad-Hoc and Cell-Based Communications". He has published over 120 papers in peer-reviewed journals and international conferences, more than 13 book chapters, and published 3 books. He served as Technical Program Committee member in major IEEE ComSoc and VTS conferences, and as the general chair of the eleventh edition of the ISWCS'2014 conference, and the co-chair of the ISWCS 2015 edition. He is IEEE Senior Member from 2007. 